\title{
NUEVAS ARAS DE CONFLOENTA, DURATÓN (SEGOVIA), HISPANIA CITERIOR, Y NUEVOS DATOS SOBRE LA INSCRIPCIÓN DE FORTUNA BALNEARIS
}

\section{NEW ALTARS FROM CONFLOENTA, DURATON (SEGOVIA), HISPANIA CITERIOR AND NEW DATA ABOUT THE INSCRIPTION OF FORTUNA BALNEARIS}

\author{
Santiago Martínez Caballero \\ Museo de Segovia \\ Juan Santos Yanguas \\ $\mathrm{UPV} / \mathrm{EHU}$
}

José Miguel Labrador

\begin{abstract}
RESUMEN. Se presentan varias aras votivas recuperadas en las excavaciones arqueológicas de las Termas Meridionales o Termas de Fortuna de Confloenta (Duratón, Segovia), Hispania Citerior, una de ellas dedicada a Fortuna, otra con texto incompleto y el resto anepígrafas. Estas piezas forman parte de un conjunto de objetos que, junto con un grupo de exvotos de terracota y piezas de vajilla, se asocian a un culto de Fortuna de carácter salutífero y curativo en este edifico de baños. Junto a ellas se da cuenta de nuevos datos sobre el epígrafe ya conocido, posible pedestal con una dedicatoria a Fortuna Balnearis, ingresada en el Museo de Segovia en los primeros días de agosto de 2020.
\end{abstract}

PALABRAS CLAVE. Confloenta, Hispania Citerior, ara, Fortuna, Fortuna Balnearis.

ABSTRACT. Some votive altars from the archaeological excavations of the Meridional Baths or Thermae of Fortuna from Confloenta (Duraton, Segovia, Hispania Citerior), are presented. One of them is dedicated to Fortuna, another one has an incomplete text and the rest are anepigraphic. These pieces are part of a set of objects that, all together with a group of terracotta votive offerings and pieces of crockery, are associated with a health and healing cult of Fortuna in this bath building. At the same time, we offer new data about an inscription, a possible pedestal with a dedication to Fortuna Balnearis, deposited in the Museum of Segovia in the first days of August 2020.

KEYWORDS. Confloenta, Hispania Citerior, altar, Fortuna, Fortuna Balnearis.

* Correspondencia a / Correspondence to: Santiago Martínez Caballero Museo de Segovia, c/ Socorro, 11 (40001 Segovia) - marcabsa@jcyl.es https://orcid.org/0000-0001-7013-2568.

Cómo citar / How to cite: Martínez Caballero, Santiago; Santos Yanguas, Juan; Labrador, José Miguel (2021), «Nuevas aras de Confloenta, Duratón (Segovia), Hispania Citerior, y nuevos datos sobre la inscripción de Fortuna Balnearis», Veleia, 38, 271-287. (https://doi.org/10.1387/veleia.22507).

Recibido: 28 enero 2021; aceptado: 30 enero 2021.

ISSN 0213-2095 - eISSN 2444-3565 / (C) 2021 UPV/EHU

cc) (i) () Esta obra está bajo una licencia

Creative Commons Atribución-NoComercial-SinDerivadas 4.0 Internacional 


\section{INTRODUCCIÓN}

Las investigaciones arqueológicas que actualmente se están desarrollando en la ciudad romana de Confloenta ${ }^{1}$, citada entre las póleis arévacas por Ptolomeo (Ptol. 2.6.55), en el conventus Cluniensis, localizada en el yacimiento arqueológico de Los Mercados, en Duratón (Segovia), han deparado el hallazgo de un pequeño grupo de aras votivas, en una de las cuales se registra el culto a Fortuna, junto a fragmentos de otras tres aras, una de ellas con una pequeña parte del texto conservado y las otras dos con el texto completamente perdido por fractura. Especialmente significativos son estos hallazgos, en primer lugar, porque todas las piezas han sido recuperadas en la excavación de las Termas Meridionales de la ciudad, en un sector muy concreto del edificio, que, a raíz precisamente de este hallazgo, ha llevado a proponer un segundo nombre a este baño público como «Termas de Fortuna» (Martínez et al. 2019a, 129). Y, en segundo lugar, porque la documentación de un testimonio de culto a Fortuna en un edifico de baños de esta ciudad de Confloenta-Duratón necesariamente ha de ponerse en relación con el conocimiento de la inscripción dedicada a Fortuna Balnearis que se conservaba empotrada en posición invertida en la fachada de un edificio de la actual población de Duratón, situada a los pies del yacimiento, conocida desde su publicación en CIL II y que ha sido muy referida y tratada en la bibliografía. Recientemente se ha derribado la vivienda y el epígrafe ha sido llevado al Museo de Segovia, de cuyos fondos forma parte inventariada como A-18.332.

La ciudad romana de Confloenta se sitúa en el alto valle del Duero, al sur de la cuenca de este río, a los pies de Somosierra, en el sector oriental del Sistema Central. Este núcleo urbano se situaba entre Termes (Tiermes, Soria) y Segovia, en el trazado de la vía que, de este a oeste, comunicaba las tierras meseteńas discurriendo paralela a la dorsal de la cordillera, muy cerca del piedemonte, y en el cruce, a su vez, con la vía que unía Complutum (Alcalá de Henares, Madrid) y Clunia (Peñalba de Castro, Burgos), atravesando en dirección norte-sur el Sistema Central, constituyendo, de hecho, Confloenta la primera ciudad en la Meseta Norte una vez superado el puerto de Somosierra ${ }^{2}$. Igualmente, la ciudad se encontraba en el paso de dos corredores pecuarios de gran importancia, integrados dentro de la red de caminos vinculados a movimientos de ganado de largo recorrido ya en época romana, incluso también prerromana ${ }^{3}$.

El centro urbano constituyó una fundación ex novo de inicios del siglo I a.C. en el ámbito suroccidental de la Celtiberia del Duero tras la conquista de este territorio en el transcurso de la Cuarta Guerra Celtibérica, acaecida durante las campañas ejecutadas por T. Didius (cos. 98 a.C.), entre 98 y 94 a.C. La nueva población surgió como la cabeza de la civitas establecida en este espacio de los valles altos de los ríos Duratón y Cega, extendiendo su territorio posiblemente también al sur del Sistema Central, en parte del alto valle del río Lozoya. El hecho se enmarca dentro de la política de urbanización romana desarrollada en este ámbito tras la conquista ${ }^{4}$, llevada a cabo por el ya procónsul Didio y la comisión senatorial de la que informa Apiano, Iber. 100, donde Con-

1 Martínez 2010 y 2014a, 130 ss.; Martínez \& Mangas 2014b; Martínez 2018; Martínez et al. 2019b.

2 Martínez 2000; Martínez 2010a; Martínez 2010b y Martínez 2014; Martínez \& Mangas 2014; Martínez 2018 y Martínez 2019, passim; Martínez, Cabañero \& Santos 2019.

3 Se trata del corredor con orientación este-oeste que, trazado paralelo y al norte de la dorsal del Sistema
Central, unía las tierras del alto Duero con el área salmantina y Extremadura, por el que luego se trazó la Cañada Real Soriana Occidental, y del corredor norte-sur que unía el alto Duero con el valle del Tajo, por donde luego se trazó la Cañada Real Segoviana. Al respecto, Martínez 2008; id. 2014, 199 ss.

4 Martínez 2011 y Martínez 2014a; Martínez et al. 2014; Santos \& Martínez 2014. 
floenta surge del traslado de la población de los oppida del entorno, principalmente la procedente de Sepúlveda (Segovia), quizás la Colenda de Apiano (Iber. 99-100), al sitio en llano de Los Mercados. El desarrollo de la ciudad dentro de este ámbito de la provincia Citerior, y desde época augustea en la Tarraconensis, llevó en época flavia a establecimiento del municipium de derecho latino, según se deduce de los documentos epigráficos 5 .

Las exploraciones arqueológicas llevadas a cabo en los últimos ańos han permitido profundizar en el conocimiento de esta ciudad, cuyo carácter urbano había quedado prácticamente olvidado hasta el inicio de los ańos 2000, en tanto que tradicionalmente se había considerado el sitio de Los Mercados como el emplazamiento de un núcleo rural o villa, a pesar de que los hallazgos registrados en las excavaciones promovidas por Manuel Godoy y acometidas en el año 1795, bajo la dirección del arquitecto real Juan de Villanueva (Martínez 2014a, 23-44), llevaron ya a J. Cornide (apud Gómez de Somorrostro 1820, 220) a plantear el carácter urbano del sitio e incluso a identificarlo con la ciudad arévaca de Confloenta. No obstante, a partir del siglo xIx el carácter urbano de Los Mercados quedaría relegado, a favor de la invención del argumento erudito de situar en la cercana Sepúlveda el sitio de una ciudad romana, intentando con ello prestigiar el pasado de esta villa de gran significado histórico en la Edad Media. El registro arqueológico documenta, en cambio, la desocupación del lugar en época romana, donde solo el lucus de Bonus Eventus, situado en las cercanías de la villa, en la margen izquierda del río Duratón, en el paraje del denominado Puente Talcano, testimonia actividad cultual: Martínez 2014a, 17 ss. Tampoco la intensa exploración de la necrópolis visigoda de Duratón entre 1942 y 1948 (Molinero 1949 y Molinero 1971), emplazada en lo que fue el suburbio de la ciudad romana, acompañada de la realización de algunos sondeos en la ciudad, llevó a reevaluar el carácter urbano de Los Mercados.

Las exploraciones arqueológicas llevadas a cabo en los años 2001 y 2002, y ya de manera sistemática desde el año 2016, están permitiendo un mejor conocimiento de la realidad urbanística de Confloenta y su desarrollo histórico. Desde el ańo 2017 uno de los objetivos de estas indagaciones está siendo la excavación de las Termas Meridionales o Termas de Fortuna, un gran edificio público de baños situado en el extremo meridional de la ciudad, construido entre fines del siglo I d.C. y el primer cuarto del siglo II d.C. ${ }^{6}$. Un edificio cuyo suministro de agua procedía de un acueducto, infraestructura de la que se conoce su existencia solo por tuberías de distribución urbana, aunque se desconoce la localización de su caput aquae y su sistema de transporte hasta la ciudad (Martínez et al. 2019a, 217-218).

Durante el transcurso de los trabajos de excavación ejecutados en estas termas se han hallado la mayor parte de aras de las que nos vamos a ocupar de inmediato, piezas que se integran con ello dentro del corpus epigráfico de la ciudad, compuesto en la actualidad por más de una treintena de piezas ${ }^{7}$. Una última ara que también analizamos fue recuperada en la excavación ejecutada por A. Molinero y L. Déroche en las termas Centrales (entonces Edificio B) en 1949 (Linage y Molinero 1986).

5 Alföldy 1977; Santos 1985; Hoyo 1995; Santos, Hoces de la Guardia \& Hoyo 2005, 81 y 78-82; Mangas 2010; Martínez 2014a, 138-145.
6 Martínez 2018, 145-147; Martínez, Cabañero \& Santos 2019, 170-179; Martínez et al. 2019a, Martínez et al. 2019b y Martínez et al. e.p.

7 Santos, Hoces de la Guardia \& Hoyo 2005, 78115; Santos \& Hoces de la Guardia 2010. 


\section{Las nuevas aras de las Termas de Fortuna}

\section{N. ${ }^{\circ}$. Ara dedicada a Fortuna (fig. 1.a y b) ${ }^{8}$}

La pieza fue hallada durante la campańa de excavaciones en las Termas Meridionales realizada en 2018, dentro de un estrato de relleno del propigneum del praefurnium principal (UE 286), que colmataba este espacio cuando ya el edifico estaba abandonado, con posterioridad al siglo iv d.C. Ingresó en el Museo de Segovia, con número de inventario DU 2018/1/286/171.

El zócalo, de sección cuadrada, presenta triple plinto escalonado, reentrantes progresivamente $2 \mathrm{~mm}$ de profundidad. Sobre el último apoya el dado epigráfico prismático, de $8,5 \mathrm{~cm}$ de anchura, $10,4 \mathrm{~cm}$ de altura y $8,6 \mathrm{~cm}$ de profundidad, de sección por tanto casi cuadrada. La cornisa, de $3,8 \mathrm{~cm}$ de altura, presenta triple moldura, con dos listeles (cada uno de $7 \mathrm{~cm}$ de altura y $5 \mathrm{~cm}$ de profundidad) que soportan un listel superior que presenta en la parte conservada $(5 \mathrm{~cm}$ de anchura), solo en la zona central frontal, $6 \mathrm{~cm}$ de altura y $5 \mathrm{~cm}$ de profundidad. No conserva la parte superior que probablemente poseería pulvini y foculus.

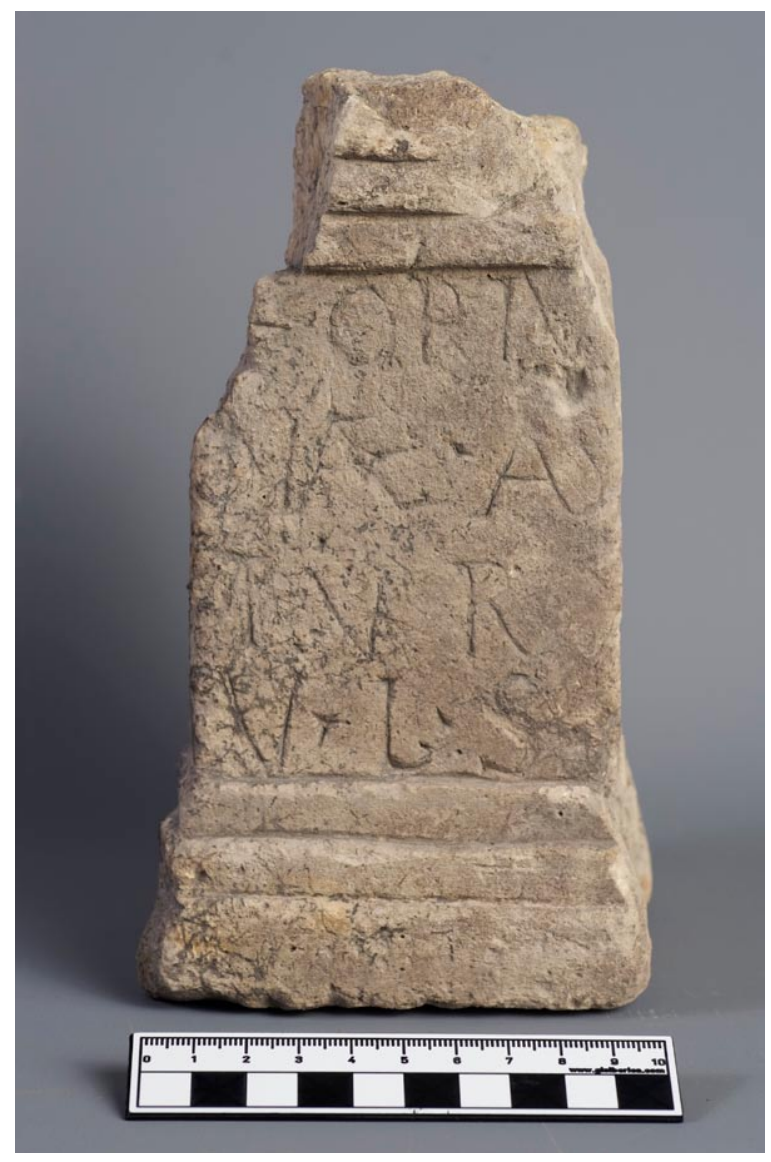

Figura 1.a. Cara anterior.

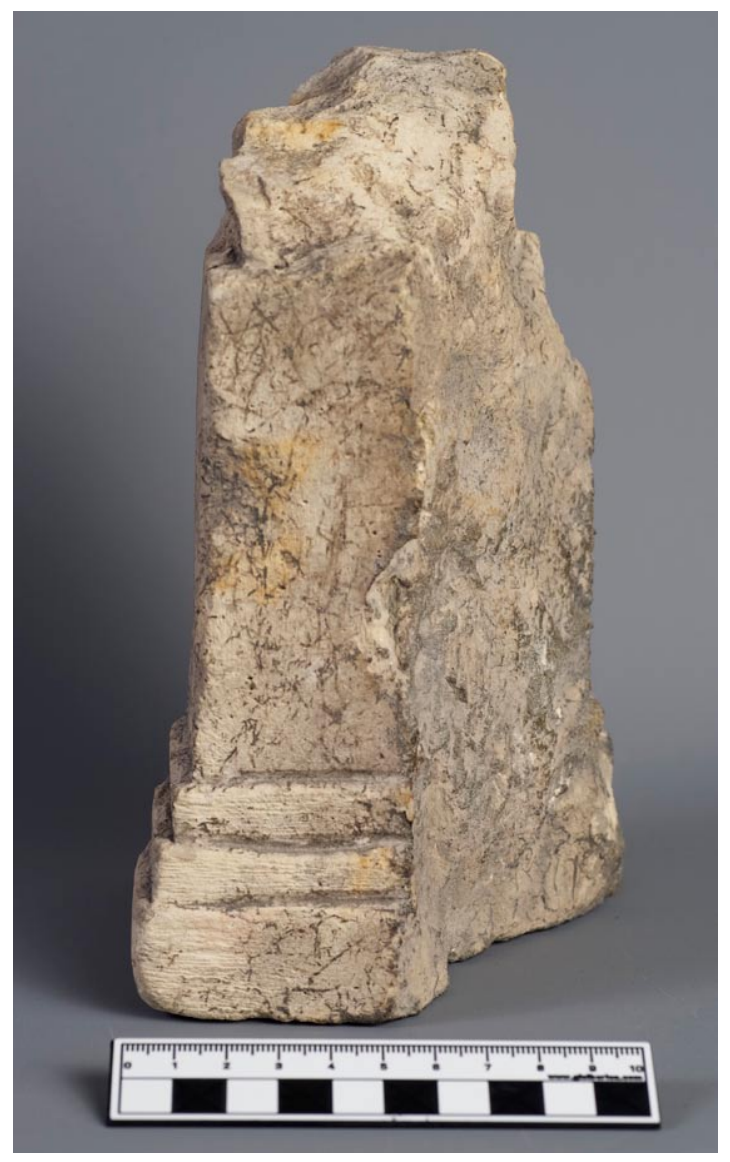

Figura 1.b. Cara lateral.

8 Todas las fotografías han sido realizadas por J. M. Cófreces Ibáñez, Museo de Segovia. 
Es habitual en las aras de Confloenta el zócalo escalonado conformado por la superposición de listeles sobre plinto, a modo de plintos superpuestos que decrecen en dimensiones, todas en aras que se datan entre inicios del siglo ir y comienzos del siglo III d.C. ${ }^{9}$. Tan solo dos aras que conservan zócalo, una en el Museo de Segovia (ERSg 112-113, n. $\left.{ }^{\circ} 44\right)$ y la pieza n. ${ }^{\circ} 4$ que presentamos en este apartado ofrecen una composición con combinación de plinto con otros tipos de molduras. En cuanto al coronamiento, la composición de listeles se vuelve a presentar en piezas de Duratón, aunque no de manera tan generalizada ${ }^{10}$, presente de nuevo en la pieza n. 5 de este apartado. La generalización de este tipo de zócalo compuesto solo con plinto y listeles es, pues, una constante en Confloenta (Hoyo, Hoces de la Guardia \& Santos 2004, 369), dato que permite hablar de un tipo de producción caracterizada por tal particularidad, que ha de responder a un hábito de talleres locales, advertido al comparar con otras árulas de la Meseta Norte (Hernández Guerra 2004) y otras áreas (Gamer 1985). Si bien podemos señalar que debe de tratarse de piezas procedentes de talleres locales, no podemos determinar si todas únicamente de uno, que hubiera estado en funcionamiento a lo largo de toda una centuria.

El texto, distribuido en cuatro líneas, está bien conservado, salvo la primera letra de línea 1, al estar roto el vértice superior del dado, fracturado siguiendo la línea del trazo vertical de la letra F. La superficie del campo epigráfico presenta múltiples arañazos, fruto, quizás, como en otros epígrafes aparecidos en este yacimiento, de las rejas del arado, y un agujero bajo la última letra de la primera línea, en la parte superior derecha.

\section{Medidas:}

Soporte: $19,2 \times 10,5 \times 10,5 \mathrm{~cm}$.

Letras: 1,$7 ; 2 ; 1,8 ; 2 \mathrm{~cm}$.

Espacios interlineales: 0,$9 ; 1 ; 0,3 \mathrm{~cm}$.

FORT^V

$\mathrm{NA} \wedge \mathrm{E} \cdot \mathrm{AS}$

TVR

$\mathrm{V} \cdot \mathrm{L} \cdot \mathrm{S}$

Transcripción: Fortu/nae As/tur / v(otum) l(ibens) s(olvit).

Traducción: A Fortuna, Astur cumplió el voto de buen grado.

Las letras corresponden a una capital rústica, irregulares y desiguales, en tamaño y trazado: $\mathrm{R}$ en primera y tercera línea; $A$ dos veces en segunda línea; $S$ en segunda y cuarta línea; y V en tercera y cuarta línea. En primera línea $\mathrm{O}$ elipsoide y $\mathrm{R}$ con óculo alto; en segunda línea, $\mathrm{S}$ en el límite del campo epigráfico y estilizada por falta de espacio; en tercera línea, $\mathrm{V}$ con trazo derecho casi vertical y R con óculo estrecho; y en cuarta línea, V con trazo derecho poco abierto. Presenta remates triangulares en algunas letras. Hay tres signos de interpunción triangulares, irregulares en tamaño.

En cuanto a la estructura del texto, la formula v(otum) l(ibens) s(olvit) no está presente en las fórmulas votivas de los epígrafes del área de la provincia de Segovia, en tanto que es habitual el or-

\footnotetext{
9 Ara de las Matres: ERSg n.o 17; ara de Minerva: ERSg n. ${ }^{\circ}$ 20; ara de Epona: Palomino et al. 2015-2016; y dos aras anepígrafas: $E R S g$ n. ${ }^{\circ} 45-46$ y pieza n. ${ }^{\circ} 7$ de este texto.
}

\footnotetext{
10 Ara de Minerva: ERSg n.o 20 y ara de Mercurius: Hoces de la Guardia, Santos \& Martínez 2015-2016.
} 
den $v$ (otum) s(olvit) l(ibens), como en las aras ERSg 17, 18, 20 (dos aras dedicadas a las Matres y una a Minerva, todas de Duratón), 58 y 59 (aras dedicadas a Arco en Saldaña de Ayllón) y 64 (ara dedicada a una deidad desconocida, pues falta el trozo superior de la inscripción en que se situaría el nombre de la divinidad, en Segovia).

La datación por paleografía lleva a una cronología del siglo in d.C., dato que concuerda bien con la datación del edificio de donde procede, construido entre fines del siglo i y el primer cuarto del siglo ir d.C., estando en uso al menos hasta inicios del siglo iv d.C.

Astur como nomen solo se registra en Bostra $(A E 1920,73)$, en la provincia de Arabia. La forma Asturius se documenta ya en el ámbito hispano en el área astur, en Villalcampo $(A E 1992,1009$; CIRPZa 243) y Gallegos del Río (CMZamora 17 = ILER 2328), en Zamora, y en el sureste, en Hellín, en Murcia ( $A E$ 1990, 626b y $A E$ 1995, 921). Como cognomen aparece en La Puebla de Trives (Ourense, CIL II, 2604 [p. 908], y CIL II, 2605) y como origo en Valencia (CIL II $2 / 14,8)$. El nombre puede señalar un origen del individuo en el área étnica astur del Noroeste hispano.

\section{N.o 2. Ara dedicada por Acca... (fig. 2, A y B)}

La pieza fue hallada en la excavación de un estrato (UE 419) que colmataba la canalización que recorre por el este el praefurnium y el alveus menor del caldarium de las Termas de Fortuna. Ingresa en el Museo de Segovia con el número de inventario DU 2019/1/419/5.

El dado epigráfico solo conserva la parte superior, con una altura de $5,8 \mathrm{~cm}$, aunque permite documentar completas su anchura de $4,8 \mathrm{~cm}$ y su profundidad de $3,7 \mathrm{~cm}$. El coronamiento, de $3,5 \mathrm{~cm}$ de altura, presenta una moldura compuesta de toro $(1 \mathrm{~cm}$ de altura y $6 \mathrm{~cm}$ de longitud en el frente), listel reentrante ( $2 \mathrm{~mm}$ de altura, $5,4 \mathrm{~cm}$ de longitud en el frente), cyme inversa (1 cm de altura y $6,8 \mathrm{~cm}$ de longitud en el frente), listel reentrante $(2 \mathrm{~mm}$ de altura y $6 \mathrm{~cm}$ de longitud en el frente) y listel $(0,9 \mathrm{~cm}$ de altura y 6,9 de longitud). Sobre el coronamiento plinto para focus de $3,1 \mathrm{~cm}$ de anchura 3,2 $\mathrm{cm}$ de profundidad y $1 \mathrm{~cm}$ de altura. La parte superior de su superficie está erosionada.

Este tipo de coronamiento más complejo remite a otras aras de Confloenta, como ERSg n. ${ }^{\circ} 87$, que también presenta cyme, entre listeles, y la n. ${ }^{\circ} 3$ de este trabajo.

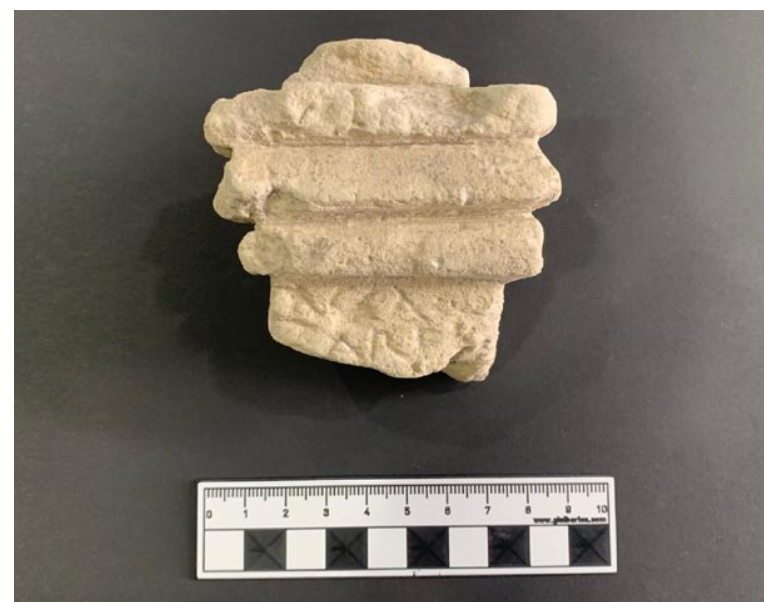

Figura 2.a. Cara anterior del ara.

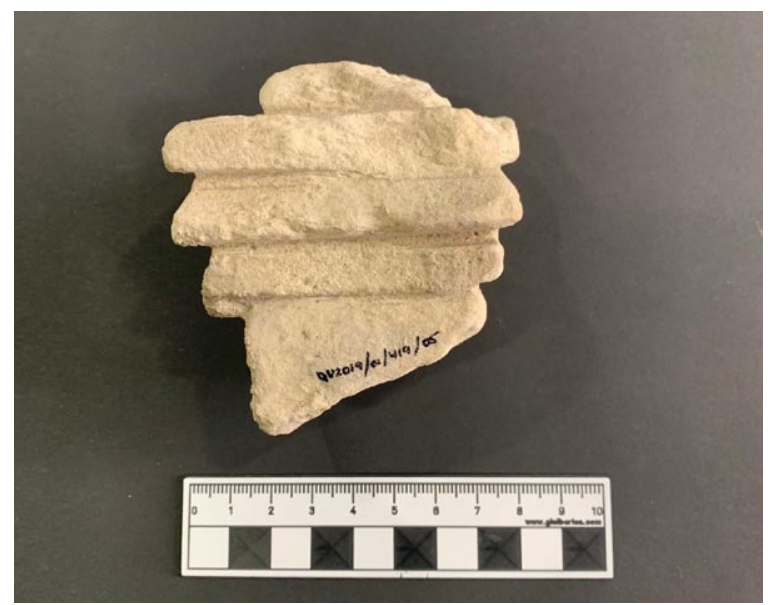

Figura 2.b. Cara posterior del ara. 
El texto solo conserva dos líneas. La línea 1 está completa, pero presenta un agujero que ha deteriorado la segunda letra y la parte superior izquierda de la primera. La segunda línea está rota en su extremo derecho, lo que ha producido la posible eliminación de una cuarta letra. En esta línea, la A puede estar antecedida de otra letra, aunque el desgaste de la pieza no permite asegurarlo. La tercera letra en línea 2 parece una $\mathrm{E}$, mejor que una $\mathrm{F}$.

Medidas:

Soporte: $7,8 \times 6,9 \times 5,1 \mathrm{~cm}$.

Letras: $7 \mathrm{~cm}$ todas.

Espacio interlineal: $2 \mathrm{~mm}$.

ACCA

[+?]ARE[+?]

Trascripción: Acca /[·]are[---].

Letra capital rústica de no muy buena factura y surcos profundos. Las tres letras A se presentan muy abiertas, la segunda de línea 1 con apex y $\mathrm{R}$ con asta muy larga.

La datación por paleografía lleva al siglo II d.C.

Acca es un antropónimo frecuente en la Celtiberia y en la Lusitania vetona, como nomen y como cognomen (Vallejo 2005, 101-103). En el territorio de Duratón se conoce una Acca Deocena Quoronicum Cadani f. (ERSg n. ${ }^{\circ}$ 61) en San Miguel de Bernuy (Segovia), aguas abajo del Duratón, a $25 \mathrm{~km}$ al noroeste de Confloenta, en el territorio de la ciudad. La secuencia «....are...» aparece en Hispania en nomina como Lareius y Vareius, y en cognomina como Bareta, Binarea, Caesarea, Careca, Careti, Caretosa, Caretus, Chares y Vare[---], de los que solo este último está documentado en áreas cercanas, en Ávila (LICS 51). Are[---], con o sin letra antes de la A, puede constituir el cognomen de $A c c a$. Como hemos indicado, parece plausible que en la segunda línea falte una letra antes de la A; en caso contrario podríamos pensar en un antropónimo del tipo Arena, (Arenna), Arenicinea, Arenia, Arentera, Arenta, Arepa, Arescusa, Ares[t]aea o Arethusa (Abascal 1994, passim), inclinándonos, por el espacio que probablemente ocupaba, por uno de los antropónimos más breves. En cualquier caso, ambos antropónimos formarían el nombre de la dedicante del ara, mujer con duo nomina.

\section{N.o 3. Fragmento de árula anepigrafa (fig. 3)}

Se trata de un fragmento del coronamiento de un árula, recuperada en uno de los estratos (UE 179) que colmataban la canalización que recorre por el este el praefurnium y el alveus menor del caldarium de las Termas Meridionales. Ingresa en el Museo de Segovia con el número de inventario DU 2018/1/179/765.

La pieza conserva parte de un ángulo y área central del coronamiento de un lateral del ara, con $5,2 \mathrm{~cm}$ de altura, $7 \mathrm{~cm}$ de anchura y $5 \mathrm{~cm}$ de profundidad. La cornisa se compone de dos toros sobre los que apoya un último listel de coronamiento, cada uno de ellos de $0,8 \mathrm{~cm}$ de altura, sobresaliendo de la vertical del dado epigráfico $1,5 \mathrm{~cm}$. Sobre el último listel se coloca un plinto $(1,2 \mathrm{~cm}$ de altura). No conserva texto. 


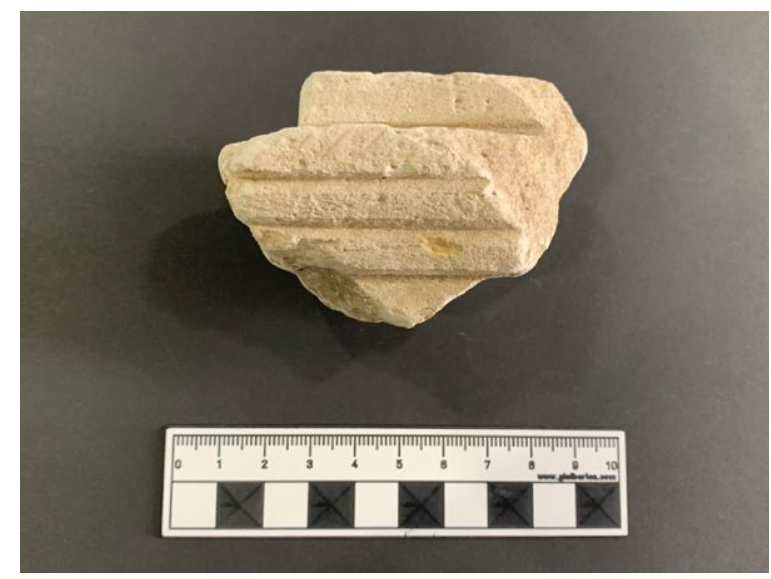

Figura 3. Fragmento de ara.

N.o 4. Fragmento de pequeña ara anepigrafa (fig. 4)

En este caso es un fragmento de un árula anepígrafa, recuperada en uno de los estratos (UE 159) que colmataban la canalización que recorre por el este el praefurnium y el alveus menor del caldarium de las Termas Meridionales. Ingresa en el Museo de Segovia con el número de inventario DU 2018/1/159/417.

La pieza conserva el coronamiento y el dado epigráfico, habíendose perdido la base. Presenta $4 \mathrm{~cm}$ de altura, $3 \mathrm{~cm}$ de anchura y $2,3 \mathrm{~cm}$ de profundidad. Sobre el dado epigráfico, sin inscripción ( $3 \mathrm{~cm}$ de altura), se coloca un coronamiento con tres listeles planos, de cerca de $0,3 \mathrm{~cm}$ de altura cada uno.

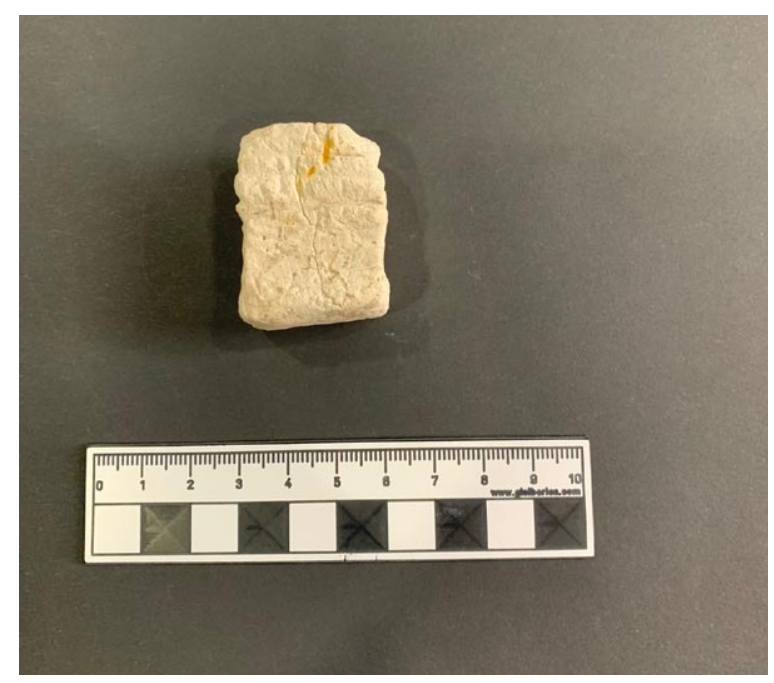

Figura 4. Cara anterior del ara. 
N.o 5. Fragmento de árula (fig. 5)

Estamos ante un fragmento de árula, recuperada en uno de los estratos (UE 85) que colmataban la canalización que recorre por el este el praefurnium y el alveus menor del caldarium de las Termas Meridionales. Ingresa en el Museo de Segovia con el número de inventario DU 2018/1/85/88.

La pieza presenta unas dimensiones de $11 \mathrm{~cm}$ de altura, $6 \mathrm{~cm}$ de anchura y $3,5 \mathrm{~cm}$ de profundidad. El dado epigráfico conserva en un cara de $7,6 \mathrm{~cm}$ de altura. Falta la otra cara completa (¿la anterior?). La cornisa se compone de dos listeles, de $2 \mathrm{~cm}$ de altura total, sobresaliendo de la vertical del dado epigráfico $0,6 \mathrm{~cm}$. Sobre el listel superior de coronamiento se coloca una pequeña parte de plinto $(1,7 \mathrm{~cm}$ de altura).

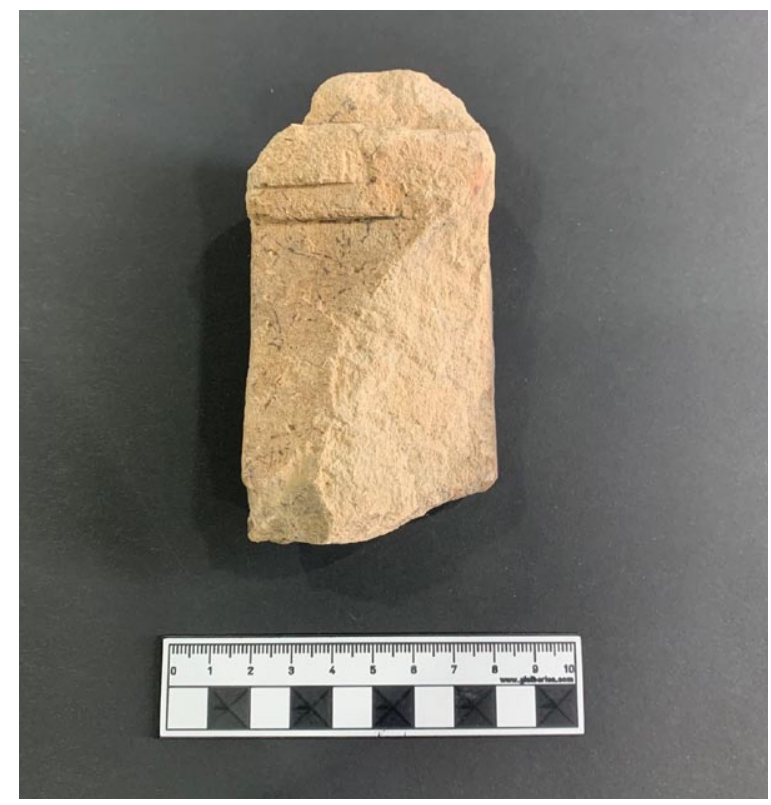

Figura 5. Árula muy fragmentada.

Como se ha señalado, la composición de listeles se vuelve a presentar en piezas de Duratón, aunque no de manera tan generalizada (ara de Minerva: ERSg n. ${ }^{\circ}$ 20, y ara de Mercurius: Hoces de la Guardia, Santos \& Martínez 2015-2016), presente también en la pieza n. ${ }^{\circ} 3$ de este apartado.

\section{N.o 6. Fragmento de árula (fig. 6.a y b)}

Se trata de fragmento de base moldurada de ara, recuperada en uno de los estratos (UE 288) que colmataban el ambiente 14, el praefurnium principal, de las Termas Meridionales, con posterioridad a su abandono. Ingresa en el Museo de Segovia con el número de inventario DU 2018/1/288/91.

La pieza no conserva ni anchura ni longitud total. En su estado la base alcanza los $14,1 \mathrm{~cm}$ de longitud, 7,8 cm de anchura y $4 \mathrm{~cm}$ de altura. Presenta un plinto de 3,2 $\mathrm{cm}$ de altura y cyme reversa de 3,4 cm de altura, sobre la que apoya el dado epigráfico. Esta base moldurada no es la más 
habitual en las aras de Duratón, aunque aparece documentada también en algunos ejemplares, según vimos al analizar la pieza n. ${ }^{\circ} 2$.

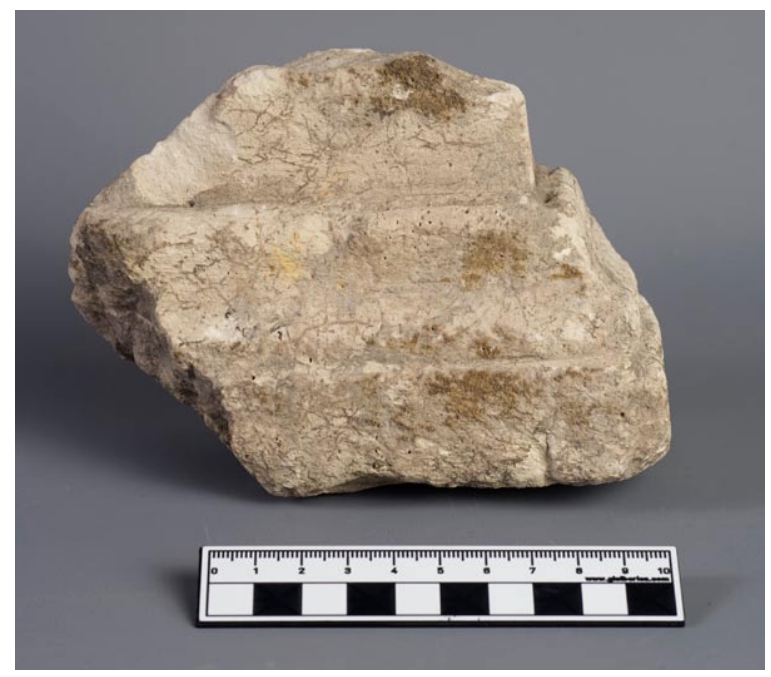

Figura 6.a. Vista frontal de la base.

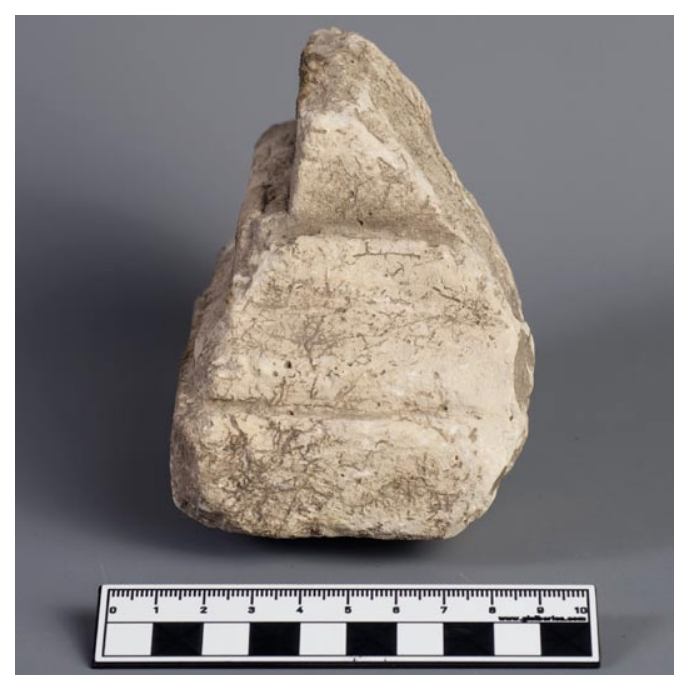

Figura 6.b. Vista lateral de la base.

De las árulas recuperadas en Confloenta, es la única que tiene en el zócalo la secuencia plinto y cyme reversa. Esa última no aparece en ninguno de los ejemplares hasta ahora conservados, como se ha señalado a propósito del ara de Fortuna (n. ${ }^{\circ} 1$ ). El dato evidencia una peculiaridad que habla de una producción menos habitual en los talleres de la ciudad, o bien que se trate de un producto de un taller no local.

\section{N. ${ }^{7}$. Fragmento de árula (fig. 7.a y b)}

Se trata de un fragmento de base de ara, recuperada en la intervención realizada por A. Molinero y L. Deroche en 1949 en Duratón (Linage \& Molinero 1986), en el entonces denominado Edificio B, ahora interpretado como vestíbulo de las Termas Centrales (Martínez 2014, 161 s.). Inventariada en el Museo de Segovia con el número A-3602.

La pieza presenta base de $10,2 \mathrm{~cm}$ de longitud, $8,8 \mathrm{~cm}$ de anchura y $6,1 \mathrm{~cm}$ de altura máximas. Ofrece el extendido modelo escalonado de zócalos de aras recuperadas en Duratón, según vimos al analizar la pieza n. ${ }^{\circ} 2$. El escalón inferior presenta $2,2 \mathrm{~cm}$ de altura, el siguiente $1,1 \mathrm{~cm}$ de altura y el tercero $0,7 \mathrm{~cm}$ de altura. Cada uno de estos escalones disminuyen progresivamente de altura de abajo hacia arriba, reentrando $0,6 \mathrm{~cm}$ cada uno con respecto a aquel en que apoyan. La parte inferior del dado epigráfico solo conserva un máximo de $2,3 \mathrm{~cm}$ de altura. 


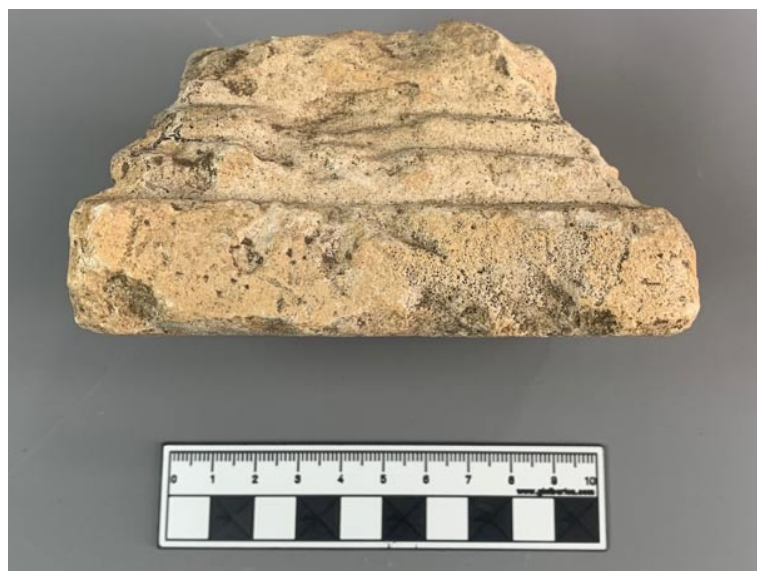

Figura 7.a. Cara anterior de la base.

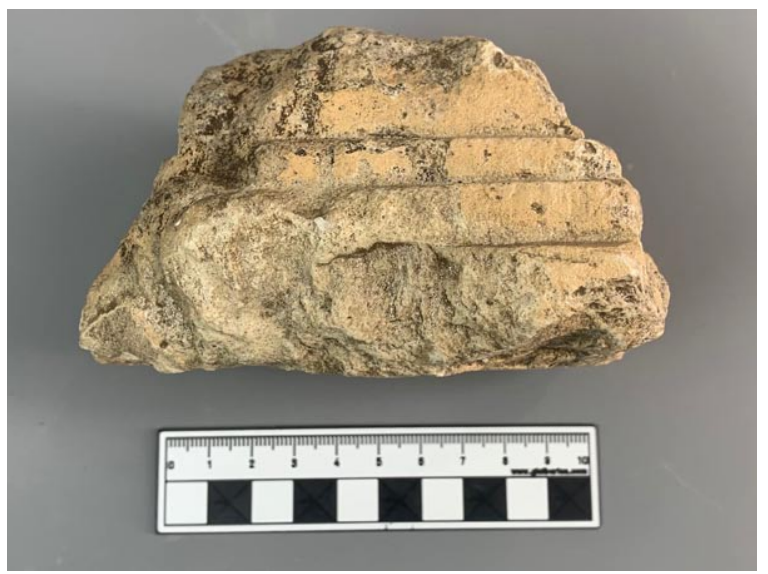

Figura 7.b. Cara posterior de la base.

\section{La insCripción de FoRTUNA BALNEARIS De Duratón (fig. 8.a, b y c)}

La dedicación del ara a Fortuna en las Termas de Fortuna constituye el segundo documento de culto a esta diosa conocido en la ciudad de Confloenta, en tanto que en el inmediato pueblo de Duratón, a $480 \mathrm{~m}$ de distancia del yacimiento arqueológico de Los Mercados, se conservaba una inscripción, bien conocida, dedicada a la Fortuna Balnearis, reutilizada como material de construcción en la fachada de una casa de esta localidad.

Con motivo del derribo de la vivienda en julio de 2020 en cuya fachada estaba incrustada, en posición invertida, se ha entregado al Museo de Segovia (n. ${ }^{\circ}$ inv. A-18.332), donde se encuentra actualmente y hemos podido estudiar sin necesidad, como en otras ocasiones, de equilibrios inseguros subidos en los peldaños de una escalera.

Se trata de un epígrafe que contiene una dedicación a la diosa realizada por un militar, Q. Valerius Bucco, soldado de la legio II Adiutrix Pia Fidelis, de la decuria de Aemilius Secundus.

El epígrafe se encontraba incrustado en la pared en posición invertida en la parte inmeditamente pegada al alero de la vivienda; probablemente esa es la razón por la que se fracturó una porción de la parte inferior, para nivelarla con el resto de la pared. Esta fractura afecta a la parte final del texto, al cerco de la cartela, formado por un marco simple y un listel liso, lo cual mide, en los tres lados que se conservan, $7 \mathrm{~cm}$. Tiene igualmente un rebaje en su cara lateral izquierda de $14 \mathrm{~cm}$, que afecta a la mitad de su superficie, realizado probablemente con la misma intención de ajustarla al muro.

Se había descrito el soporte como bloque (ERSg n. ${ }^{\circ} 16$ ), pero, una vez extraído de la fachada de la casa de la calle de la Ronda s/n de Duratón, donde se encontraba, y habiendo podido realizar su análisis directo en el Museo de Segovia, probablemente debamos hablar de un pedestal con unas medidas originales de $60 \times 40 \times 40 \mathrm{~cm}$. Se llega a esta medida de la altura sumando a los $47 \mathrm{~cm}$ conservados los 7 del marco y el listel exteriores y los probables $5 / 6 \mathrm{~cm}$ de las letras de la supuesta línea 9 perdida, que analizaremos más adelante. Por estar únicamente labrada la cara anterior en que se encuentra la inscripción podemos deducir que, en su situación original, debió estar pegado al muro, quizás con una representación de la diosa (no sabemos si busto o estatua) en su parte superior. 


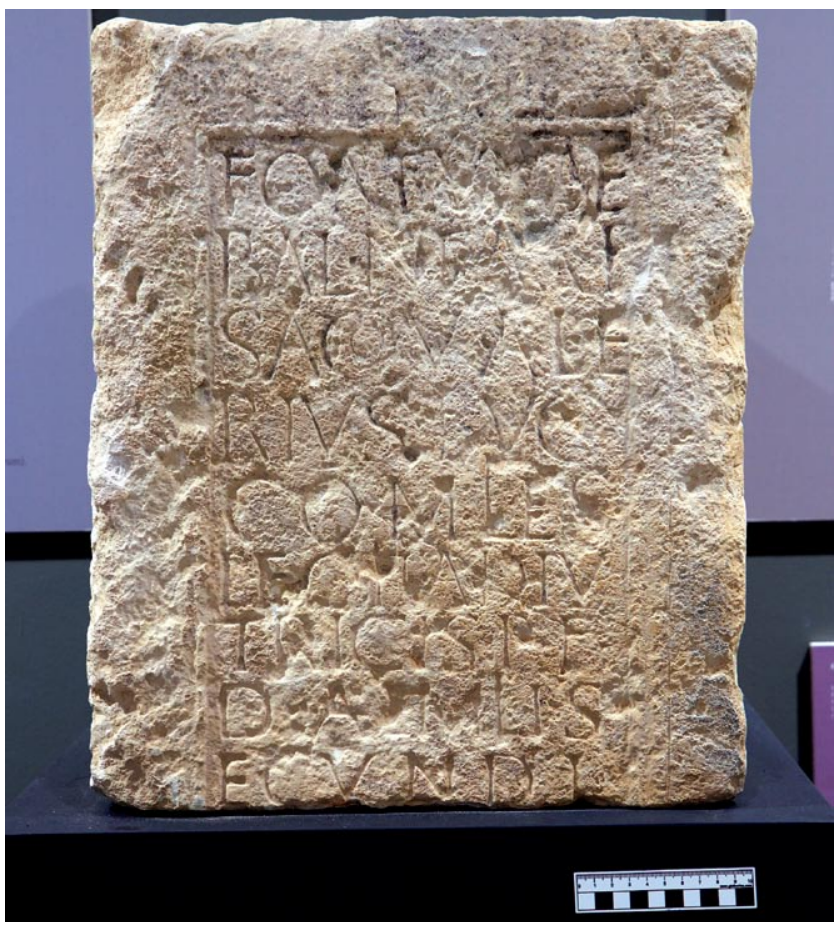

Figura 8.a. Cara anterior inscrita.

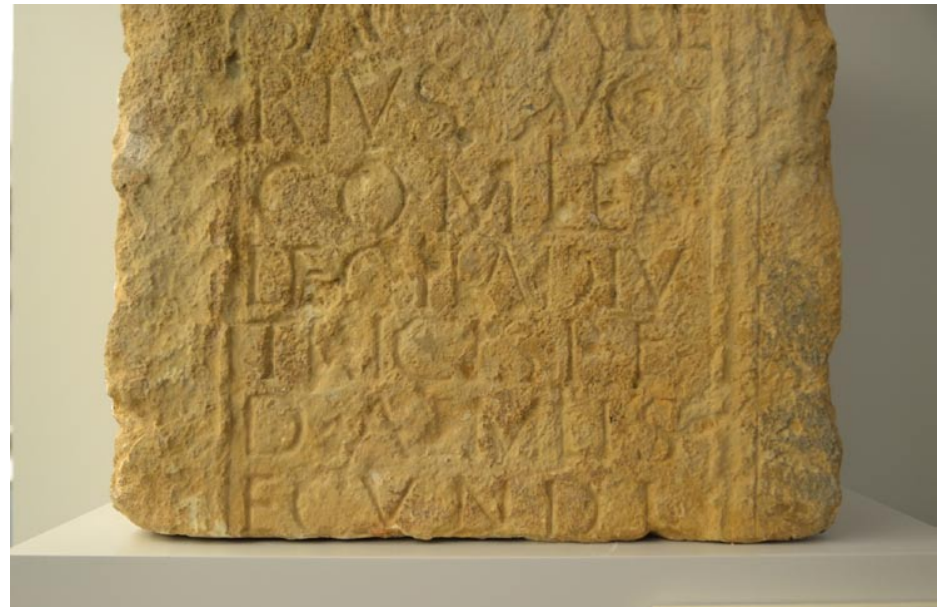

Figura 8.b. Detalle de la parte inferior.

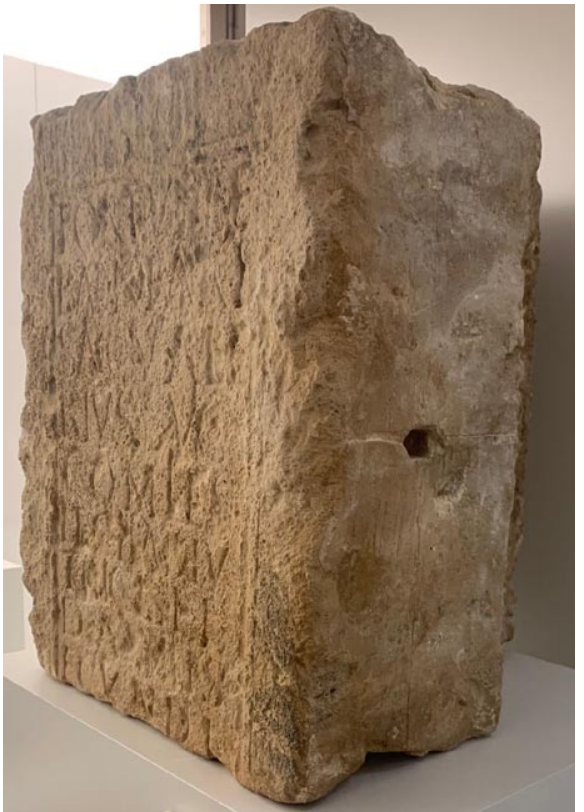

Figura 8.c. Cara lateral derecha. 
Medidas:

Soporte: $(47) \times 39,6 \times 40$

Campo epigráfico: $(40) \times 25 \mathrm{~cm}$.

Letras: 4; 4; 4,3; 4; 4 (el nexo LI 5); 3,8/4; 3,8; 3,8; $3,8 \mathrm{~cm}$.

Espacios interlineales: 0,$8 ; 06 ; 0,7 ; 0,8 ; 0.4 ; 0,5 ; 0,4 ; 0,8 \mathrm{~cm}$.

El texto presenta la siguiente lectura:

FORTV[N]AE
BALNEARI
SAC Q VALE
RIVS BUC
CO MILES
LEG II ADIV
TRICIS P F
D AMEILI S
ECVNDI
[-----]

Tanscripción: Fortu[n]ae / Balneari / sac(rum) Q(uintus) Valelrius Buclco miles / leg(ionis) II Adiul tricis P(iae) F(idelis) / D(ecuriae) Aemili(i) S/ecundi / -.---.

Traducción: Consagrado a Fortuna Balnear. Quinto Valerio Bucón, soldado de la legión II Adiutrix Pia Fidelis, de la decuria de Emilio Segundo ....

Las variaciones en diferentes lecturas propuestas por autores precedentes se recogen en ERSg (82-84, n. ${ }^{\circ}$ 16): línea 3 SA (hedera) Hernández, SAC (hedera) Knapp; línea 4, TVC y LVC CIL, TVC Arribas y Conte y Fernández; línea 8, D · AEMIIS Anonymus, D · AEMILI · S Hernández; D(OMITI?) Arribas; por D CIL y sus dependencias y Knapp. También algunos autores, comenzando por Roldán (1974, n. ${ }^{\circ}$ 725), han interpretado (centuriae) Aemili (el último Andreu 2018), aunque claramente se lee (y más ahora que se puede hacer una cómoda autopsia) D.

El texto presenta letra capital cuadrada de buena factura y regular, con excelente paginación, si no fuera porque las letras finales de líneas 1,2 y 3 están grabadas parcialmente sobre el marco. Presenta signos de interpunción triangulares y nexos IL en líneas 5 y 8.

Del análisis directo de la pieza, de su ubicación anterior en la parte superior de la fachada inmediatamente debajo del alero y del contenido de la misma nos atrevemos ahora a proponer la desaparición por rotura de una línea final en la que iría la fórmula de dedicación del tipo V.S.L.M o EX VOTO, en ambos casos con una paginación con justificación central. Probablemente, si nos inclinamos por pensar que se trata de un pedestal, la fórmula más adecuada podría ser ex voto.

Quizás el análisis onomástico de Q. Valerius Bucco pueda proporcionarnos algún dato sobre su origen y su presencia en Duratón. Su nomen es Valerius, el segundo más extendido en Hispania (Abascal 1994, 232-244; OPEL IV, 142-146), siendo muy habitual en la Celtiberia y el primero en la provincia de Segovia y en Confloenta y su territorio. En la vecina Segovia, Valerius se documenta hasta en dieciséis ocasiones. Bucco es conocido tanto en Hispania, como fuera de ella (Kajanto 1982, 63 y 208; OPEL I, 325; Vallejo 2005, 139). R. C. Knapp (1992, 291) argumenta para este militar un origen cisalpino, aunque no hay argumentos definitivos. Se ha señalado también que, en base a su onomástica, Q. Valerius Bucco podría tener un origen peregrino y liberto, 
siendo precisamente la Legio II Adiutrix formada en Ravenna (se señala como responsable a $\mathrm{Mu}$ cianus) con marineros desertores de Vitelio en 69 d.C., quienes, como era habitual, tenían un origen peregrino o servil, señalándose que la decuria de la que formaba parte podría estar integrada por los primeros decuriones de la legión, a la que pudiera haber pertenecido este militar, de rango desconocido (Illarregui 2015, 108). Se ha planteado la posibilidad de la presencia de la Legio II Adiutrix en la última década del siglo I d.C., en sustitución de la Legio VII Gemina, durante su estancia temporal en Germania. Es muy probable que de este momento proceda la estancia de este militar en Confloenta. El dato concordaría con una cronología de la primera mitad del siglo iI d.C. en base a la mención del nombre completo de la legio (Le Roux 1982, 159), y con la colocación de la dedicatoria en los primeros momentos de uso de las Termas de Fortuna, a inicios del siglo II d.C.

\section{Abreviaturas}

AE = Année Epigraphique.

CIL II = Corpus Inscriptionum Latinarum.

$C I R P Z a=$ Corpus de inscripciones romanas de la provincia de Zamora. Fuentes epigráficas para la historia social de Hispania romana.

CMZamora = Catálogo Monumental de España. Provincia de Zamora.

$E R S g=$ J. Santos Yanguas, Á. L. Hoces de la Guardia \& J. Del Hoyo, 2005, Epigrafía romana de Segovia y su provincia.

$H E p=$ Hispania Epigraphica .

$I L E R=$ Inscripciones Latinas de la España romana.

LICS = R. C. KNAPP, 1992, Latin Inscriptions of Central Spain.

$O P E L=$ B. LörinCz \& F. Redö, Onomasticon Provinciarum Europae Latinarum.

\section{BibLiOgRAFÍA}

Abascal Palazón, J. M., 1994, Los nombres personales en las inscripciones latinas de Hispania [Anejos de Antigüedad y Cristianismo II], Murcia: Universidad Complutense de Madrid, Universidad de Murcia.

AlföLdy, G., 1977, «Eine römische Inschrift aus Duratón in der Hispania Citerior», Zeitschrift für Papyrologie und Epigraphik 27, 222-228.

Alonso Ávila, A., \& S. Crespo Ortiz de Zárate, 1999, Las manifestaciones religiosas del mundo antiguo en Hispania romana: el territorio de Castilla y León. I: Las fuentes epigráficas, Valladolid: Edición de los autores.

Andreu Pintado, J., 2018, «La sacralización del agua en Hispania romana, una perspectiva epigráfica», en: M. J. Pérex Agorreta, C. Miró y Alaix (eds.), Ubi aquae ibi salus: aguas biomedicinales, termas curativas y culto a las aguas en la Peninsula Ibérica (desde la Protohistoria hasta la Tardoantigüedad), Madrid, Universidad Nacional de Educación a Distancia, 91-120.

Arribas Clemente, M. E., 1983, Fuentes de Segovia Antigua, Memoria de Licenciatura inédita, Universidad Complutense de Madrid.

Conte Bragado, D., \& I. Fernández Bernaldo de Quirós, 1993, Introducción a la arqueología en el Cañón del Duratón, Segovia: Diputación Provincial de Segovia.

Crespo Ortiz de Zárate, S., 1978, «Segovia y la sociedad de época romana. Las fuentes epigráficas», Durius $6,11-12,179-219$. 
Crespo Ortiz de Zárate, S., 1984, «Fuentes epigráficas para el estudio de las formas religiosas en el conventus Cluniensis durante la época romana», en: L. A. de Cuenca, E. Gangutia Elícegui, A. Bernabé Pajares, J. López Facal (coords.), Athlon. Satura Grammatica in honorem Francisci R. Adrados II, Madrid: Gredos, 191-224.

Gamer, G., 1989, Formen römischer Altäre auf der Hispanischen Halbinsel, [Madrider Beiträge; Band 12] Mainz am Rheim: Verlag Philipp von Zabern.

Gómez de Somorrostro, A., 1820, El Acueducto y otras antigüedades de Segovia, Madrid: Imprenta de D. Miguel de Burgos.

Hernández Guerra, L., 2004, «Pequeños altares en el área de la Meseta Septentrional», Hispania Antiqua XXVIII, 153-168.

Hoces de la Guardia Bermejo, Á. L., J. Santos Yanguas \& S. Martínez Caballero, 2015-2016, «Ara votiva de Confloenta (Duratón, Segovia), dedicada a Mercurius», Segovia Histórica 2-3, 9-15.

Hoyo, J. DEL, 1995, «Duratón, municipio romano. A propósito de un fragmento inédito de ley municipal», Zeitschrift für Papyrologie und Epigraphik 108, 140-144.

Hoyo, J. Del, 2000, «¿Santuario dedicado a Diana cercano a Sepúlveda (Segovia)?», Veleia 17, 135-141.

Hoyo, J. del, Á. L. Hoces de la Guardia Bermejo \& J. Santos Yanguas, 2004, «Taller de aras votivas de Duratón", en: L. Hernández Guerra, J. Alvar Ezquerra (eds.), Actas del XXVII Congreso Internacional GIREA-ARYS IX, "Jerarquias religiosas y control social en el mundo antiguo", (Valladolid, 7 al 9 noviembre 2002), Valladolid: Universidad de Valladolid, 367-370.

Illarregui, E., 2015, «Ara a Fortuna Balnearis de Duratón (Segovia, España)», Oppidum. Cuadernos de investigación 11, 99-112.

Kajanto, I., 1982, The Latin Cognomina, Roma: Giorgio Bretschneider Editore.

Knapp, R. C., 1992, Latin Inscriptions of Central Spain, Berkeley: Univ. of California Press.

Le Roux, P., 1981, «Inscriptions militaires et déplacemets de troupes dans l'empire romaine», Zeitschrift für Papyrologie und Epigraphik 43, 195-206.

Le Roux, P., 1982, L'Armée romaine et l'organisation des provinces ibériques d'August à l'invasion de 409, $\mathrm{Pa}-$ ris: Diffusion de Boccard.

Le Roux, P., 2011, La toge et les armes. Roma entre Méditerranée et Océan, Rennes: PU Rennes (Histoire).

Linage Conde, A., \& A. Molinero Pérez, 1986, «Unas excavaciones inéditas de 1949 en el pueblo de Duratón, Sepúlveda», en: Homenaje a Pedro Sainz Rodríguez, T. I, Madrid: Fundación Universitaria Española, 235-245.

LörInCZ, B., 2002, Onomasticon Provinciarum Europae Latinarum, vol. IV: QVADRATIA-ZVRES, Viena.

LörIncz, B., \& F. Redö, 1994, Onomasticon Provinciarum Europae Latinarum, vol. I: ABA-BYSANVS, Budapest.

Mangas Manjarrés, J., 2010, "Ciudades y municipios del ámbito segoviano en época romana», en: S. Martínez Caballero, J. Santiago, A. Zamora (coords.), Segovia romana II. Gentes y territorios. Segovia: Obra Social Caja Segovia, 123-142.

Martínez Caballero, S., 2000, «Notas sobre la romanización del territorio segoviano», en: VV.AA., Segovia romana, Segovia: Caja de Ahorros de Segovia, 11-42.

Martínez Caballero, S., 2008, «Los territorios de los municipios del Sur del Conventus Cluniensis (Hispania Citerior) en el Alto Imperio: Termes, Duratón y Segovia», en: J. Mangas, M. Á. Novillo (eds.), El territorio de las ciudades romanas, Madrid: 187-247.

Martínez Caballero, S., 2010a, «La ciudad de Confluentia-Duratón y su territorio», en: S. Martínez Caballero, J. Santiago, A. Zamora (coords.), Segovia romana II. Gentes y territorios. Segovia: Obra Social Caja Segovia, 183-219.

Martínez Caballero, S., 2010b, «Los territorios segovianos entre la conquista romana y el fin de la República», en: S. Martínez Caballero, J. Santiago, A. Zamora (coords.), Segovia romana II. Gentes y territorios. Segovia: Obra Social Caja Segovia, 39-74.

Martínez Caballero, S., 2011, «La ciudad fundada por M. Marius, Termes y Colenda (App., Iber. 99-100). La guerra de 104-93 a.C. en territorios arévacos, vacceos y vettones», SH. Historia Antigua 29, 119-151. 
Martínez Caballero, S., 2014, Confloenta, la ciudad romana de Duratón. La historia, la ciudad, el territorio, los cultos, Segovia: Diputación Provincial de Segovia.

Martínez Caballero, S., 2018, «La ciudad romana de Confloenta (Duratón, Segovia). El urbanismo», en: S. Martínez Caballero, J. Santos Yanguas, L. J. Municio González (coords.), El urbanismo de las ciudades romanas del valle del Duero. Actas de la I Reunión de Ciudades Romanas del Valle del Duero [Anejos de Segovia Histórica 2], Segovia: Junta de Castilla y León, Diputación de Segovia, Ayuntamiento de Segovia y Asociación de Amigos del Museo de Segovia, 137-156.

Martínez Caballero, S., \& J. Mangas Manjarrés, 2014, «Evolución urbana y difusión de los modelos arquitectónicos romanos en la ciudad de Confloenta (Duratón, Segovia, Hispania Citerior)», en: J. M. Álvarez Martínez, T. Nogales, I Rodà (eds.), Actas XVIII Congreso Internacional de Arqueología Clásica. Centro y periferia en el mundo clásico. Mérida, 13-17 de Mayo de 2013, Mérida: Museo Nacional de Arte Romano, 71-75.

Martínez Caballero, S., V. M. Cabañero Martín \& J. Santos Yanguas, 2019, «Segovia en el Alto Imperio: los siglos I y II d.C.», en: S. Martínez Caballero (coord.), Historia de Segovia. Volumen 2. La Antigüedad, Segovia: Diputación de Segovia, 99-383.

Martínez Caballero, S., F. López Ambite \& J. I. Gallego Revilla, 2014, «Tiermes y el área arévaca occidental (siglos IV a.C.-I d.C.). La Protohistoria como modelo de frontera», en: F. Burillo Mozota (ed.), M. Chordá (coord.), VII Simposio sobre celtíberos. Nuevos hallazgos, nuevas interpretaciones (Daroca, 20-22 de marzo de 2012), Teruel: Centro de Estudios Celtibéricos de Segeda, 93-102.

Martínez Caballero, S., C. Martín García, J. M. Labrador Vielva, V. M. Cabañero Martín \& J. Resino Toribio, 2019a, «Las Termas Meridionales y las Termas Centrales de Confloenta (Duratón, Segovia)", en: Congreso Internacional de termas públicas de Hispania, Murcia, 19-21 de abril de 2018, Murcia: Universidad de Murcia.

Martínez Caballero, S., C. Martín García, J. M. Labrador Vielva, V. M. Cabañero Martín \& J. Resino Toribio, 2019b, "Confloenta (Duratón). Una ciudad romana en la Meseta norte», en: E. Gamo Pazos, J. Fernández Ortega, D. Álvarez Jiménez (eds.), En ningún lugar... Caraca y la romanización de la Hispania interior, Guadalajara: Diputación de Guadalajara, 467-493.

Martínez Caballero, S., C. Martín García, J. M. Labrador Vielva, V. M. Cabañero Martín \& J. Resino Toribio, e. p., "Las Termas Meridionales de Confloenta (Duratón, Segovia», en: S. Martínez Caballero, J. Santos Yanguas, V. M. Cabañero Martín, L. J. Municio González (coords.), Actas del Coloquio de la I Reunión de Arqueología Clásica de Segovia, 17 y 18 de octubre de 2019, Segovia.

Molinero Pérez, A., 1949, «La necrópolis visigoda de Duratón (Segovia): Materiales de tipo bizantino», Crónica del IV Congreso Arqueológico del Sudeste Español (Elche, 1948), Cartagena, 497-505.

Molinero Pérez, A., 1971, Aportación de las excavaciones y hallazgos casuales (1941-1959) al Museo de Segovia [Excavaciones Arqueológicas en España 71], Madrid: Ministerio de Cultura.

Palomino Lázaro, Á., J. M. Gonzalo González, S. Martínez Caballero, J. Santos Yanguas \& Á. L. Hoces de la Guardia Bermejo, 2015-2016, «Nueva ara votiva de Confloenta (Duratón, Segovia), dedicada a la diosa Epona», Segovia Histórica 2-3, 17-23.

Perea Yébenes, S., 1997, «Baños para soldados y el culto a Fortuna», en: M. J. Pérex (ed.), Termalismo Antiguo. I Congreso Peninsular. Actas, Madrid: Universidad Nacional de Educación a Distancia, 149-167.

Roldán Hervás, J. M., 1974, Hispania y el ejército romano, Salamanca: Universidad de Salamanca.

Sagredo San Eustaquio, L., \& A. Jiménez de Furundarena, 1996, «La religión practicada por los militares del ejército romano de Hispania durante el Ato Imperio Romano (siglos I-III)», Espacio, Tiempo y Forma. Serie II, Historia Antigua 9, 289-319.

Santos Yanguas, J., 1985, «La inscripción de Puente Talcano, Sepúlveda, Segovia (CIL II 5095=3089). Nueva lectura e interpretación», en: J. L. Melena (ed.), Symbolae L. Mitxelena septuagenario oblatae [Anejos de Veleia 1], Vitoria: Universidad del País Vasco / Euskal Herriko Unibertsitaea, 537-545.

Santos Yanguas, J., Á. L. Hoces de la Guardia \& J. Del Hoyo, 2005, Epigrafía romana de Segovia y su provincia. Segovia: Caja de Ahorros de Segovia, Diputación de Segovia. 
Santos Yanguas, J., \& S. Martínez Caballero, 2014, «Modelos de urbanización republicana en la Meseta Norte oriental: la Celtiberia de arévacos y pelendones», en: M. Chiabà (ed.), Hoc quoque laboris praemium. Scritti in onore di Gino Bandelli, Trieste: Università di Studi di Trieste, 457-476.

Solana Sainz, J. M., \& L. Hernández Guerra, 2000, Religión y sociedad en época romana en la Meseta Septentrional, Valladolid: Universidad de Valladolid.

Vallejo, J. M.a, 2005, Antroponimia indigena de la Lusitania romana, Anejos de Veleia, Series Minor 23, Vitoria-Gasteiz: Servicio Editorial de la Universidad del País Vasco / Euskal Herriko Unibertsitatearen Argitalpen Zerbitzua. 